\title{
Spontaneous duodenocutaneous fistula: a rare complication of duodenal ulcer
}

\author{
A.N. Reddy \\ University of Nevada School of Medicine and the V.A. Medical Center, 1000 Locust Street, Reno, Nevada \\ 89520, USA.
}

\begin{abstract}
Summary: Fistula formation between the duodenum and the skin of the anterior abdominal wall is a rare complication of duodenal ulcer disease, most often felt to occur following ulcer surgery. The development of a spontaneous duodenocutaneous fistula in association with duodenal ulcer has not been hitherto reported. A middle aged man with a history of peptic ulcer for more than 10 years, and a vagotomy and pyloroplasty 15 months earlier, presented with an asymptomatic duodenocutaneous fistula, presumably secondary to a perforated duodenal bulb ulcer. Successful healing of the fistula was accomplished by non-surgical therapy consisting of nasogastric suction, intravenous alimentation and parenteral cimetidine over a 4-week period.
\end{abstract}

\section{Introduction}

Duodenal ulcer is an unusual cause of duodenocutaneous fistula. Most such fistulae are thought to occur in an immediate postoperative period and are felt to be iatrogenic (vide infra). A review of the previous publications has failed to disclose any case of spontaneous duodenocutaneous fistula as a complication of duodenal ulcer disease. The purpose of this paper is to report such a case, review the literature pertinent to the subject and emphasize the importance of non-surgical management in healing the fistula.

\section{Case report}

A 51 year old Caucasian man presented with a history of having developed a fistula in the upper abdomen approximately 3 weeks earlier. He had denied any history of antecedent abdominal trauma, inadvertent ingestion of sharp foreign bodies, abdominal pain, nausea, vomiting, fever or jaundice. The drainage fluid was described as being odourless, clear and light yellow in colour and free of any visible blood or pus, but the patient may have noticed some food particles on occasion.

The patient gave a history of peptic ulcer disease exceeding 15 years and had undergone simple

Correspondence: A.N. Reddy, M.D., F.A.C.G., F.A.C.P., 129 West Sixth Street, Suite No. 2, Reno, Nevada 89503 Accepted: 27 October 1987 closure of a perforated duodenal ulcer 19 years previously. He remained symptom free for the subsequent 8 years, but then developed massive upper gastrointestinal haemorrhage requiring suture-ligation of the bleeding gastroduodenal artery in the base of a duodenal ulcer. Two weeks later (approximately 15 months before the current hospitalization for the fistula) a truncal vagotomy and pyloroplasty were done as definitive therapy for his duodenal ulcer disease. Following the last operation the patient had remained symptom free until the onset of the fistula.

Physical examination revealed a thin-built man with a $5 \mathrm{~mm}$ fistulous opening located $2 \mathrm{~cm}$ below and to the right of the xiphoid, at the upper end of a well healed paramedian surgical incision from previous abdominal operations, draining light yellow transparent fluid. The rest of the patient's physical examination was normal.

The routine laboratory data were all within normal range. Quantitation of the fistulous drainage yielded approximately $200 \mathrm{ml}$ per day of clear, odourless bilious fluid, which, unfortunately, was not tested for $\mathrm{pH}$ or amylase. However, Gram stain and cultures of the fistulous drainage yielded no pathogens. A serum gastrin determination was within normal limits. A fistulogram using an iodinated contrast material showed that the fistula was in communication with the pyloroduodenal region (Figure 1). Barium contrast radiographic studies revealed a deformed pyloroduodenum with

(C) The Fellowship of Postgraduate Medicine, 1988 


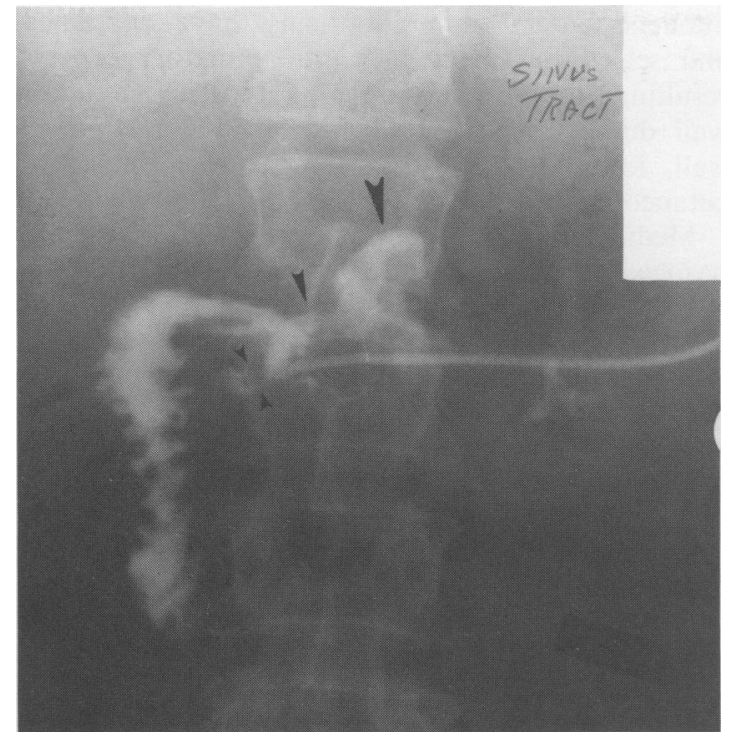

Figure 1 Fistulogram reveals the tip of the catheter located in the antero-inferior wall of the duodenal bulb where an ulcer-like projection with oedematous collar can be seen (small arrows). The medium arrow points to the pyloroduodenal junction, which is obviously deformed. The large arrow points to the gastric antrum. Incidentally, the vagotomy surgical clip is seen at the left upper corner of T-12 vertebral body.

an anterior wall fistula, and normal small and large intestines.

Subsequently, the patient underwent an oesophagogastroduodenoscopy which revealed a markedly distorted pyloroduodenum, with retained sutures (from previous pyloroplasty). The duodenal bulb was seen to be occupied by the balloon of the catheter passed through the external opening of the fistula for drainage purposes. Upon removal of the balloon catheter the duodenal bulb revealed a 5$6 \mathrm{~mm}$ opening in the anterior wall with friable and inflamed margins but without nodularity or a mass lesion. By an external inspection of the patient's anterior abdominal wall at this juncture, one could see the tip of the endoscope to lie subjacent to the external fistulous opening, thus confirming the presence of duodenocutaneous fistula. Biopsies from the fistulous opening in the duodenal bulb, taken to exclude the presence of unsuspected Crohn's disease or malignancy, yielded acute and chronic inflammation without granulomas.

As the diagnosis was being established, the patient received nothing by mouth, nasogastric suction, and intravenous glucose and electrolytes, but this was changed after the diagnosis, to intravenous hyperalimentation (using hypertonic dextrose, aminoacid and lipid solutions). Cimetidine, $300 \mathrm{mg}$, was administered intravenously every 6 hours to decrease the output of gastric acid. During the subsequent 4 weeks the amount of the fistulous drainage progressively decreased and finally stopped with healing of the external fistulous opening. A follow-up upper gastrointestinal barium $\mathrm{X}$-ray study revealed a $1.5 \mathrm{~cm}$ tract ending blindly in a pseudodiverticulum which emanated from the anterior wall near the base of the duodenal bulb (Figure 2). Oral feedings were gradually resumed after 4 weeks of the above management and the patient was maintained on oral cimetidine for an additional period of 6 months. When last seen, approximately 3 years from the above episode, for an unrelated medical problem, the patient was free of any gastrointestinal symptoms.

\section{Discussion}

Fistulization between the duodenum and the anterior abdominal wall is a rare complication of duodenal ulcer, with little or no information on the subject being found in the major gastrointestinal and standard surgical text books. ${ }^{1-5}$ Besides the well known complications of an ulcer, namely, free

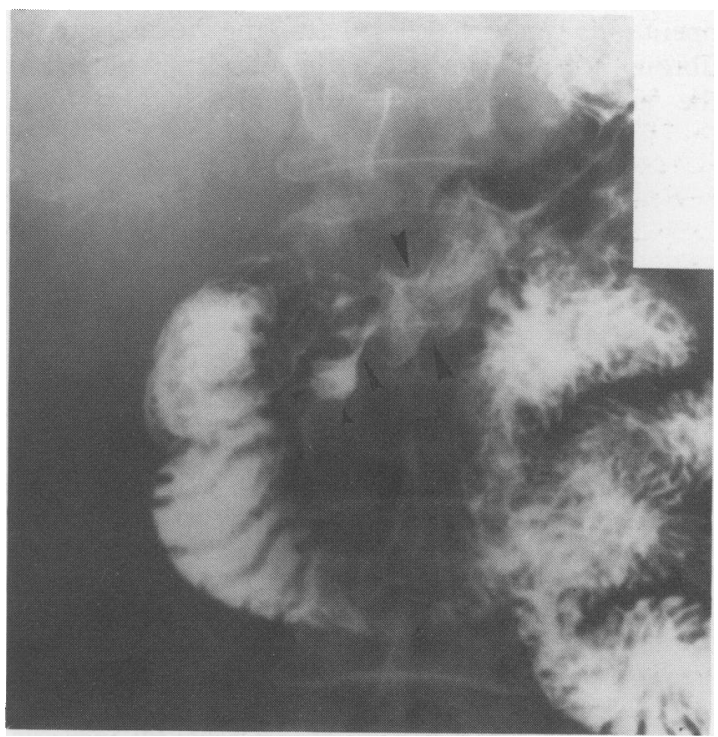

Figure 2 Barium-meal study performed 4 weeks after medical therapy reveals the remnant of the healed duodenocutaneous fistulous tract ending as a blind pseudodiverticulum (small arrows) that seems to emanate from the antero-inferior wall of the deformed duodenal bulb (medium arrow). The large arrows mark the pyloroduodenal junction. 
perforation with peritonitis, haemorrhage, posterior penetration and obstruction, a variety of confined perforations into different anatomical structures have been described with respect to gastric and duodenal ulcers. These include ulcer penetration into the pancreas, gastro-hepatic omentum, transverse mesocolon, liver, gall bladder, bile duct, transverse colon, retroperitoneum, subhepatic space and rarely into the portal vein, pleura or even pericardium. ${ }^{1,6,7}$ Tarazi et al. in their review of 47 cases of gastric and duodenal cutaneous fistulae found that the majority of the fistulae $(70-90 \%)$ were felt to be secondary to operations performed on duodenum or -stomach and were considered to be iatrogenic. ${ }^{8}$ Other aetiologies included trauma, inflammatory processes, ischaemia and radiationinduced injury. In another series, Fitzgibbons et al. have described seven cases of duodenal Crohn's disease, two of whom had duodenal cutaneous fistulae requiring combined medical and surgical treatment. ${ }^{9}$ However, neither of the series had reported any cases of spontaneous duodenocutaneous fistula, related to duodenal ulcer disease.

In contrast to the reviewed cases of Tarazi et al., 8 the subject of this report had his last surgery approximately 15 months previously, thus making any direct causal relation to the subsequent development of a fistula less likely. It is conceivable, however, that the three previous ulcer operations may have led to the formation of fibrous adhesions between the duodenal bulb and the anterior abdominal wall. Presumably, these

\section{References}

1. Pelot, D. \& Hollander, D. Complications of peptic ulcer disease. In: Berk, K.E. (ed) BockusGastroenterology, 4th ed., vol. 2. W.B. Saunders Co., Philadelphia, 1985, pp 1155-1185.

2. Walker, C. Complications of peptic ulcer disease and indications for surgery. In: Sleisenger, M.H. and Fordtran, J.S. (eds) Gastrointestinal Disease, 3rd ed. W.B. Saunders Co., Philadelphia, 1983, pp 725-738.

3. Reber, H.A. Abdominal abscesses and gastrointestinal fistulas. In: Sleisenger M.H. and Fordtran, J.S. (eds) Gastrointestinal Disease, 3rd ed. W.B. Saunders Co., Philadelphia, 1983, pp 319-335.

4. Moody, F.G., McGreevy, J.M. Stomach. In: Schwartz, S.I., Shires, G.T., Spencer, F.C. and Storer, E.H. (eds) Principles of Surgery, 4th ed. McGraw-Hill Book Co., New York, 1984, pp 1113-1145.

5. Gastric and duodenal fistulas and perforations. In: Shackelford, R.T. and Zuidema, G.D. (eds) Surgery of the Alimentary Tract, 2nd ed. vol. 2. W.B. Saunders Co., Philadelphia, 1981, pp 270-276. duodeno-parietal adhesions may have contained and selectively directed the inflammatory process resulting from perforation of a recurrent anterior wall duodenal bulb ulcer towards the abdominal wall, leading to the development of a duodenocutaneous fistula.

Medical management, consisting of nil by mouth, nasogastric suction and parenteral alimentation, successfully closed the fistula in our patient, similar to the results of a third of Tarazi's patients. ${ }^{8}$ However, our patient also received cimetidine to reduce the output of acid and pepsin, a point stressed by Feller et al. in their management of choledochoduodenal fistula due to a penetrating peptic ulcer. ${ }^{6}$ The role of intravenous hyperalimentation in the non-surgical management of gastrointestinal fistulae in general is controversial, ${ }^{3,8,9}$ although this may have played a beneficial role in our subject.

In summary, this is the first reported case of a spontaneous duodenocutaneous fistula due to confined perforation of an anterior mass duodenal ulcer that closed successfully after medical therapy. The presumed pathogenesis of the lesion, its medical management and a review of the pertinent literature have been presented.

\section{Acknowledgement}

The author would like to thank Ms Frieda Hill for her secretarial help in preparation of this manuscript.
6. Feller, E.R., Warshaw, A.L. \& Schapiro, R.H. Observations on management of choledochoduodenal fistula due to penetrating peptic ulcer. Gastroenterology 1980, 78: 126-131.

7. Michowitz, M., Farago, C., Lazarovici, I. \& Solowiejczyk, M. Choledochoduodenal fistula: a rare complication of duodenal ulcer. Am J Gastroenterol 1984, 79: 416-420.

8. Tarazi, R., Coutsoftides, T., Steiger, E. \& Fazio, V.W. Gastric and duodenal cutaneous fistulas. World J Surg 1983, 7: 463-473.

9. Fitzgibbons, T.J., Green, G., Silberman, H., Eliasoph, J., Halls, J.M. \& Yellin, A.E. Management of Crohn's disease involving the duodenum, including duodenal cutaneous fistula. Arch Surg 1980, 115, 1022-1028. 